\title{
A conformational switch in the DiGIR1 ribozyme involved in release and folding of the downstream I-Dirl mRNA
}

\author{
HENRIK NIELSEN, ${ }^{1}$ CHRISTER EINVIK, ${ }^{2}$ THOMAS E. LENTZ, ${ }^{1}$ MADS MARQUARDT HEDEGAARD, ${ }^{1}$ \\ and STEINAR D. JOHANSEN ${ }^{3}$ \\ ${ }^{1}$ Department of Cellular and Molecular Medicine, The Panum Institute, University of Copenhagen, DK-2200N Copenhagen, Denmark \\ ${ }^{2}$ Department of Pediatrics, University Hospital of North Norway, N-9038 Tromsø, Norway \\ ${ }^{3}$ Department of Molecular Biotechnology-RNA Research Group, Institute of Medical Biology, University of Tromsø, N-9037 Tromsø, Norway
}

\begin{abstract}
DiGIR1 is a group I-like cleavage ribozyme found as a structural domain within a nuclear twin-ribozyme group I intron. DiGIR1 catalyzes cleavage by branching at an Internal Processing Site (IPS) leading to formation of a lariat cap at the 5' -end of the $3^{\prime}$ cleavage product. The $3^{\prime}$-cleavage product is subsequently processed into an mRNA encoding a homing endonuclease. By analysis of combinations of $5^{\prime}$ - and $3^{\prime}$-deletions, we identify a hairpin in the $5^{\prime}$-UTR of the mRNA (HEG P1) that is formed by conformational switching following cleavage. The formation of HEG P1 inhibits the reversal of the branching reaction, thus giving it directionality. Furthermore, the release of the mRNA is a consequence of branching rather than hydrolytic cleavage. $A$ model is put forward that explains the release of the I-Dirl mRNA with a lariat cap and a structured 5'-UTR as a direct consequence of the DiGIR1 branching reaction. The role of HEG P1 in GIR1 branching is reminiscent of that of hairpin P-1 in splicing of the Tetrahymena rRNA group I intron and illustrates a general principle in RNA-directed RNA processing.
\end{abstract}

Keywords: Didymium iridis; group I intron; DiGIR1 branching ribozyme; conformational switching

\section{INTRODUCTION}

RNA molecules in general have the ability to adopt many different conformations. This ability is played out during folding of the RNA into the biologically relevant conformation that frequently follows one or more distinct pathways in which mutually exclusive base pairings replace each other (Thirumalai et al. 2001; Woodson 2002; Schroeder et al. 2004). Transcriptional order plays an important part in this process because sequence stretches become available for folding in an order that does not necessarily favor direct folding into the active conformation. Thus, in many cases alternative conformations of an RNA molecule are simply a consequence of the ordered synthesis by its transcription and sequential association with, e.g., protein factors. In some cases, the ability to adopt alternative conformations plays a functional role. This can be linked to transcription, e.g., as a way of activating the biologically relevant conformation, or it can be a post-transcriptional ability of the RNA to undergo a

Reprint requests to: Henrik Nielsen, Department of Cellular and Molecular Medicine, The Panum Institute, University of Copenhagen, 3 Blegdamsvej, DK-2200N Copenhagen, Denmark; e-mail: hamra@sund. ku.dk; fax: +4535327732.

Article published online ahead of print. Article and publication date are at http://www.rnajournal.org/cgi/doi/10.1261/rna.669209. conformational switching as part of the function of the particular RNA molecule. Examples of conformational switching are known from different molecular processes including replication of viruses (Badorrek et al. 2006), regulation of transcription and translation in prokaryotes by riboswitches (Tucker and Breaker 2005), and the functions of the ribosome (Rodnina and Wintermeyer 2003) and the spliceosome (Konarska et al. 2006). A subclass of RNA conformational switches has been described as self-induced because the functional transition is determined by the RNA itself without the involvement of other molecules (Nagel and Pleij 2002). Here, the RNA is folded into a metastable structure, which is maintained for some time, allowing or blocking a particular function of the RNA. When a different sequence element subsequently becomes available for base pairing, e.g., by transcription or a processing reaction, this triggers the conformational switching that allows the RNA to adopt a different conformation that has a functional property distinct from that of the starting conformation. Examples of such self-induced switches comprise group I introns, the hepatitis delta virus ribozyme, the RNA genome of coli-phage MS2, and the hok/sok system of plasmid R1 from Escherichia coli (Nagel et al. 2002).

The concept of self-induced conformational switching provides important clues to the function of the complex 
twin-ribozyme group I introns. These introns are composed of a conventional group I splicing ribozyme (GIR2) with an insertion into a peripheral loop of a cassette composed of the branching ribozyme GIR1 and a downstream homing endonuclease encoding sequence (for review, see Johansen et al. 2002, 2007; Nielsen et al. 2008). Examples of twin-ribozyme introns are currently limited to the extrachromosomal rDNA of the myxomycete Didymium iridis (Dir.S956-1) and several species and lineages of the amoebaflagellate Naegleria (Nae.S516) (Nielsen et al. 2008). In the twin-ribozyme intron Dir.S956-1, DiGIR2 is responsible for splicing, reverse splicing, and circularization of the intron, and DiGIR1 is responsible for cleavage at an internal processing site (IPS) within the intron to form the $5^{\prime}$-end of the I-DirI mRNA (Decatur et al. 1995; Einvik et al. 1998). The DiGIR1 cleavage occurs by a transesterification reaction and is initiated by nucleophilic attack of the $2^{\prime}-\mathrm{OH}$ of an internal nucleotide (U232) at the IPS. As a result, the $5^{\prime}$-product containing the core of the ribozyme has a $3^{\prime}-\mathrm{OH}$, and the $3^{\prime}$-product is left with a small lariat at the $5^{\prime}$-end in which the first and the third nucleotides are linked by a 2', 5' - phosphodiester bond (Nielsen et al. 2005; Beckert et al. 2008). This reaction is referred to as the branching reaction throughout this paper.

We have previously noted that nucleotides taking part in the formation of key elements (Fig. 1, left panel, P2, P10) in the catalytically active form of DiGIR1 could alternatively engage in the formation of a hairpin (Fig. 1, right panel, HEG P1), also present in the $5^{\prime}$-UTR of the released I-Dir I mRNA (Vader et al. 1999; Einvik et al. 2000). The helical structures $\mathrm{P} 2$ and $\mathrm{P} 10$ are supported by mutagenesis analysis (Einvik et al. 1998, 2000), and our recent modeling of DiGIR1 based on comparison to the Azoarcus splicing ribozyme demonstrates a role for P10 in formation of the active site (Beckert et al. 2008). Thus, formation of HEG P1 is expected to result in an inactive conformation of DiGIR1. HEG P1 apparently plays a dual role during processing of the Dir.S956-1 intron. (1) Prior to splicing catalyzed by DiGIR2, HEG P1 forms as part of an inactive form of DiGIR1 as evidenced by structure probing (M. Hedegaard and $\mathrm{H}$. Nielsen, unpubl.). In contrast to the splicing ribozymes, DiGIR1 has to fold into an inactive conformation during transcription in order not to inactivate the rRNA precursor by cleavage. (2) Following splicing and DiGIR1-catalyzed branching, HEG P1 forms as part of a structured $5^{\prime}$-UTR of the I-DirI mRNA. In the present study, we report that the cleavage rate and end-point of the reaction as well as the cleavage mechanism (branching versus hydrolysis) of length variants of the DiGIR1 ribozyme are critically dependent on the relative stability of two alternative structures. Furthermore, we show that the release of the mRNA from the ribozyme core and the folding of the 5'-UTR of the mRNA are directly coupled to the branching reaction by an RNA conformational switch.

\section{Active}

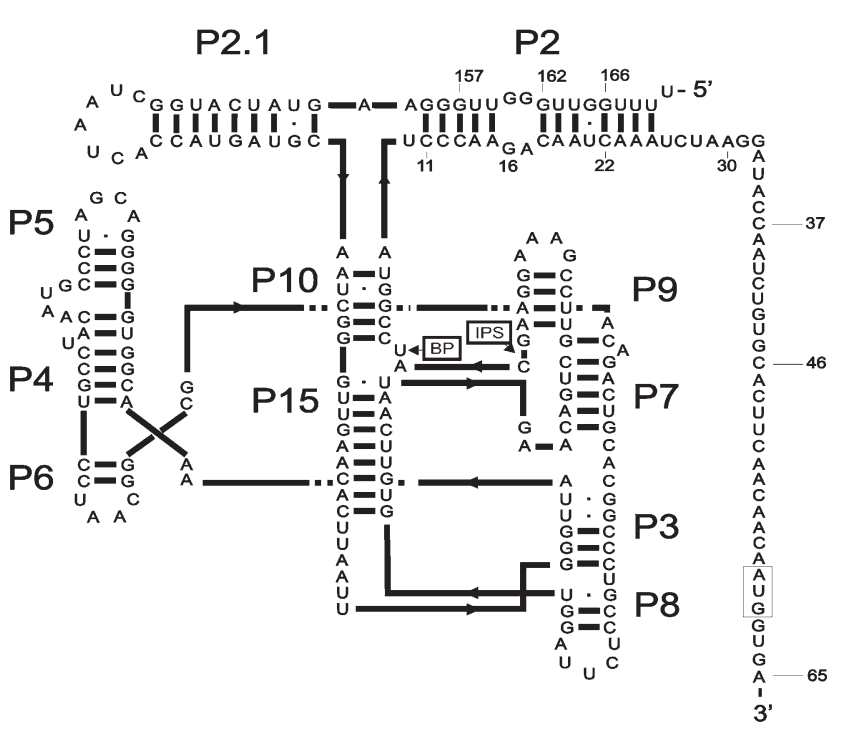

\section{Inactive}

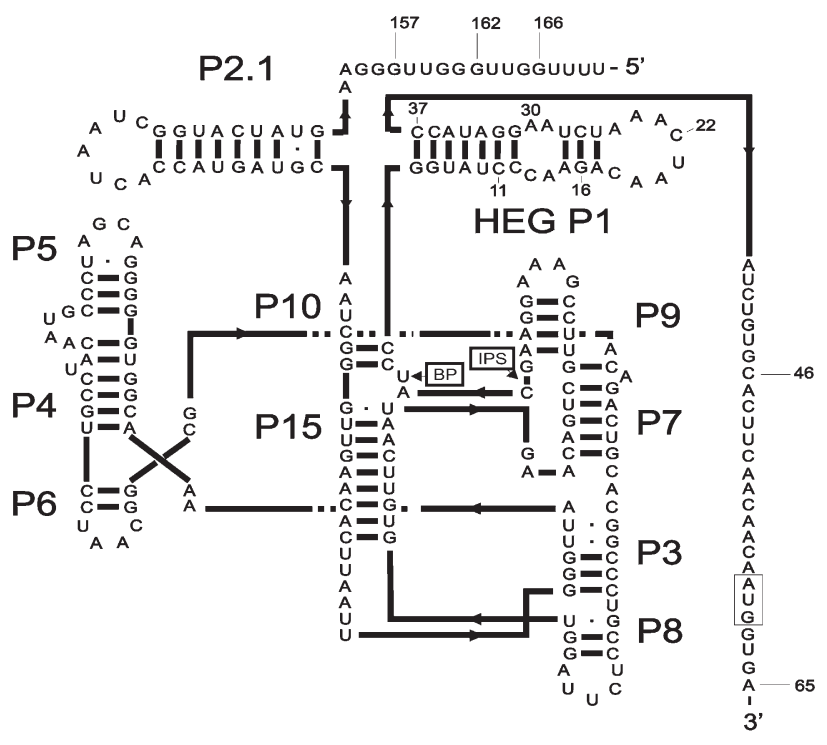

FIGURE 1. Helix diagrams of the catalytically active conformation of DiGIR1 compared with an alternative, inactive conformation. The difference between the two is the engagement of $\mathrm{P} 10^{\prime \prime}$ and $\mathrm{P} 2^{\prime \prime}$ (the $3^{\prime}$-strands of the two helices) of the catalytically active conformation in formation of HEG P1 in the inactive conformation. HEG P1 is also found as a structural element in the $5^{\prime}$-UTR of the I-DirI mRNA. It is possible that the inactive conformation has additional structural differences from the active conformation than HEG P1 emphasized in the figure. The branch point (BP) and internal processing site (IPS) are indicated, and the initiation codon of the HE open reading frame is boxed. The numbers indicate the positions upstream or downstream, respectively, in relation to the IPS. These positions mark the number of nucleotides included in the deletion constructs analyzed in the paper. 


\section{RESULTS}

\section{Ligation is inhibited by the formation of HEG P1}

In the study of ribozymes, a proper delimitation of model ribozymes is a major issue. The question of reversibility of the reaction is related to this because the forward and reverse reactions can be differently affected by deletion of flanking sequences. We previously noted that cleavage of DiGIR1166.22 (carrying 166 nucleotides [nt] upstream of IPS, and $22 \mathrm{nt}$ downstream from IPS) by branching was highly reversible, in contrast to cleavage by DiGIR1-157.22 (Nielsen et al. 2005). In Figure 2A, we outline all of the reactions that have been experimentally demonstrated previously. These are cleavage by transesterification (Fig. 2A, "1A"), the reverse of this reaction (ligation) (Fig. 2A, "1B"), and cleavage by hydrolysis (Fig. 2A, "2A"). Cleavage by transesterification (branching) is the resulting reaction in vivo (Vader et al. 1999; Decatur et al. 2000), and cleavage by hydrolysis is considered an in vitro artifact (Nielsen et al. 2005). To expand on the importance of the ligation reaction in cleavage analyses, a ligation experiment with $3^{\prime}$-fragments of varying length was carried out. The $3^{\prime}$-fragments were gel purified from body-labeled cleavage reactions of 157.X length variants that are known to cleave predominantly by the branching reaction and to be poor in ligation. The 5 '-fragment was isolated from an unlabeled cleavage reaction of 166.65. The resulting reaction in this case is hydrolysis, and the $5^{\prime}$-fragment is known to carry out efficient ligation when mixed with a 22-nt 3 '-fragment (Nielsen et al. 2005). In Figure 2B, almost no ligation product is seen to accumulate in the negative control reaction $157+22$. In contrast, a ligation product is immediately apparent after mixing of $166+22$. The amount of ligated product decreases with time of incubation. Primer extension analysis of the timecourse samples revealed that this was due to subsequent hydrolytic cleavage of the ligated product, 166.22, as expected (data not shown). The products of the hydrolysis reaction are unable to re-ligate, explaining the decrease in ligated product over time in the experiment. Importantly, in the ligation reactions with $3^{\prime}$-fragments of varying lengths, very little ligation product is formed when $\geq 37 \mathrm{nt}$ are included in the $3^{\prime}$-fragment. This is not due to rapid turnover of the ligated product because no change in primer extension pattern was observed in the time-course samples (data not shown). An alternative approach to elucidate the influence of HEG P1 on ligation is an experiment with 65-nt 3 '-fragments carrying mutations in HEG P1. HEG P1mut1 is a 2-nt substitution in the internal loop, and HEG P1mut2 is a disruption of the entire hairpin (Fig. 2C). The $3^{\prime}$-fragments were isolated from body-labeled cleavage reactions of 157.65HEG P1mut 1 and 157.65HEG P1mut2 that proceed predominantly by branching (data not shown). In order to isolate the ligation component of the reactions, the forward reaction by branching was inhibited by using an acidic ligation buffer (Johansen and Vogt 1994; Einvik et al. 1998). As seen in Figure 2C, the wild-type (wt) ligation reaction was very slow, whereas the reaction with a disrupted HEG P1 (mut2) went to completion in a few minutes. The reaction with the mutant affected in the internal loop (mut1) was slightly better than wt, arguing that the internal loop contributes to the stability of the hairpin or inhibits the ligation in other ways. From these experiments, we conclude that the formation of HEG P1 inhibits the ligation reaction and thus contributes to the directionality of the DiGIR1 branching reaction.

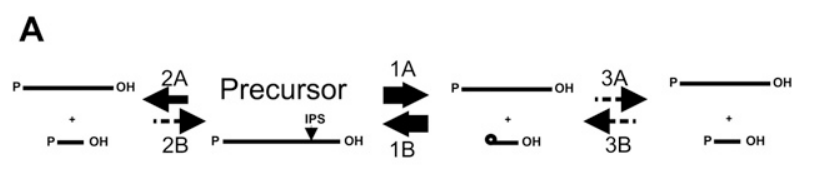

B

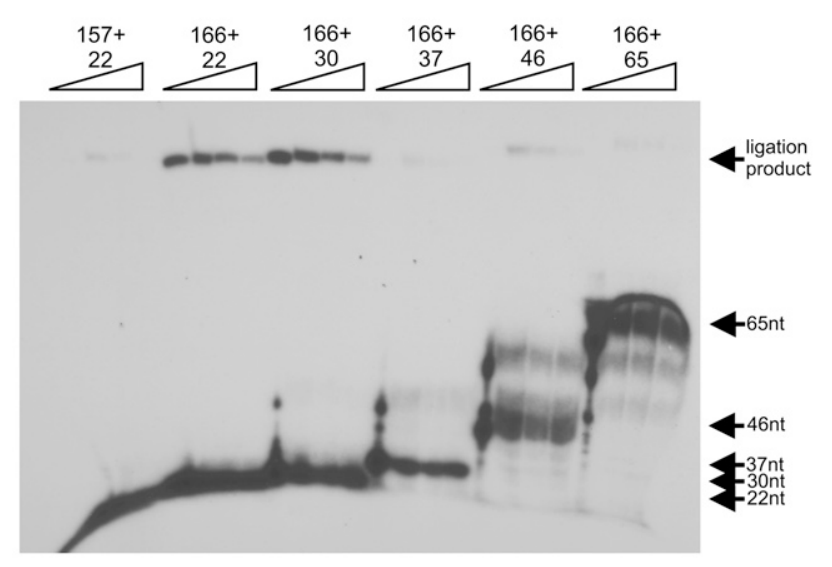

C
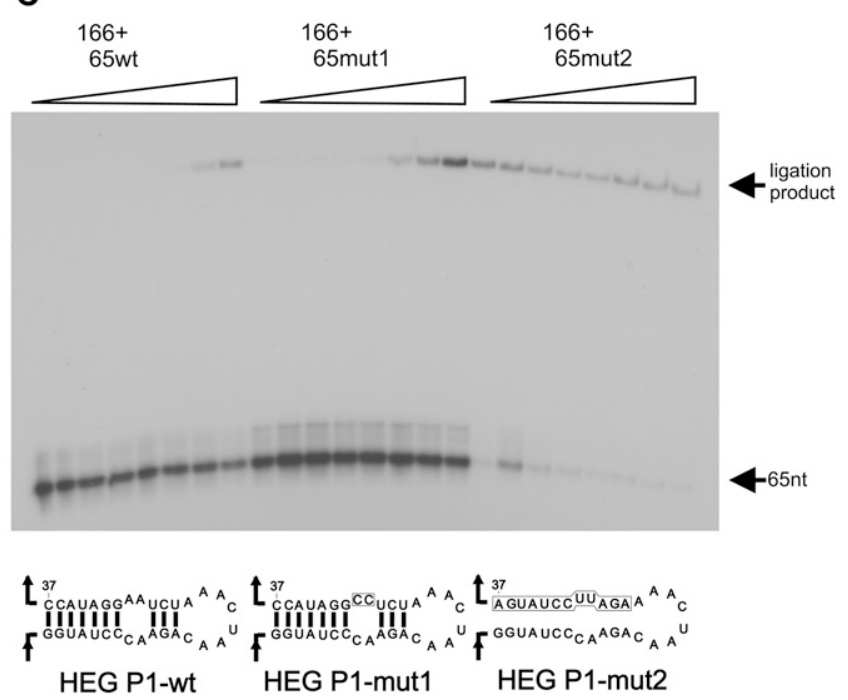

FIGURE 2. (Legend on next page) 


\section{Several aspects of DiGIR1 cleavage are affected by $5^{\prime}$ - and 3 '-flanking sequences}

In light of the results from the ligation experiments, we systematically evaluated the effects of varying the length of the $3^{\prime}$-end in three different $5^{\prime}$-end sequence contexts (157 nt, $162 \mathrm{nt}$, and $166 \mathrm{nt})$. In addition to kinetic cleavage analyses, we used primer extension analysis to discriminate between cleavage by branching that results in a primer extension pause signal at the branch point (BP) and cleavage by hydrolysis that results in a primer extension stop signal due to run-off at the IPS. Our initial study of $5^{\prime}$ - and $3^{\prime}$ deletions in DiGIR1 concluded in the choice of construct DiGIR1-162.65 as a model due to the relatively high $k_{\text {obs }}$ observed (Einvik et al. 2000). Primer extension analysis in this case showed a pause at the BP similar to that of cellular RNA (Vader et al. 1999), and contrasting with the predominant primer extension stop at IPS found in analysis of the full-length intron (Johansen and Vogt 1994; H. Nielsen, unpubl.). We concluded that this construct better reflected the in vivo ribozyme than the full-length intron when tested in vitro (Einvik et al. 2000). The original $3^{\prime}$-deletion analysis is reproduced in Figure $3 \mathrm{~B}$ and shows a gradual decrease in $k_{\text {obs }}$ and end-point values with shortening of the $3^{\prime}$-end. We have now tested the same series of $3^{\prime}$-end deletions in a construct that contains an additional $4 \mathrm{nt}$ at the $5^{\prime}$-end (166.X) and a construct with a further shortening of $5 \mathrm{nt}$ (157.X) (the $5^{\prime}$ - and $3^{\prime}$-coordinates are indicated in Fig. 1 and the $k_{\mathrm{obs}}$ and end-point values are listed in Supplemental Table 1). In the 157.X series (Fig. 3C), the short variants have the highest observed cleavage rates and the lowest end points and group together. The long variants all have a lower observed cleavage rate and a significantly higher end point. The differences between the two groups, in particular with respect to end-point values, coincide with deletion of the sequence between nucleotides 30 and 37 downstream from IPS. This sequence corresponds to the 3 '-strand of HEG

FIGURE 2. (A) Reactions that are considered in the interpretation of the cleavage analyses: (1A) branching reaction in which the precursor is cleaved at IPS, resulting in a $5^{\prime}$-product with a $3^{\prime}-\mathrm{OH}$ and a $3^{\prime}-$ product with a $5^{\prime}$-lariat cap in which the first and the third nucleotides are joined by a 2', 5'-phosphodiester bond (Nielsen et al. 2005); (1B) reversal of the branching reaction; (2A) cleavage at the IPS by hydrolysis resulting in a $5^{\prime}$-product with a $3^{\prime}-\mathrm{OH}$ and a $3^{\prime}$-product with a $5^{\prime}$-phosphate; (2B) reverse of the hydrolytic cleavage reaction; (3A) hydrolytic opening of the lariat; (3B) formation of the lariat directly from the $5^{\prime}$-cleavage product. The thickness of the arrows indicates the efficiency of the isolated reactions. (Dashed arrows) Reactions that have not been observed in the present experiments. (B) Influence of HEG P1 on ligation. Ligation of unlabeled 166-nt $5^{\prime}$-cleavage fragment with labeled $3^{\prime}$-cleavage fragment of variable lengths. Time samples were withdrawn at $t=0,2,15$, and 60 min. $(C)$. Effect of mutation of HEG P1 on ligation. The experiment was conducted similarly to the experiment described above except that an acidic ligation buffer was used in order to inhibit the forward reaction and that more time samples were withdrawn for analysis $(t=$ $0,1,2,5,10,30,60$, and $120 \mathrm{~min}$ ). The effect of the mutations on the base pairing potential in HEG P1 is shown below the gel picture.
P1 (Fig. 1B). Consistent with this, the mutant construct DiGIR1-157.65mut2, in which the sequence of the 3 '-strand of the hairpin is replaced with its complementary sequence, has much higher $k_{\text {obs }}$ than DiGIR1-157.65. In fact, the $k_{\text {obs }}$ of this mutant is close to that of DiGIR1-162.65wt (data not shown). When the $3^{\prime}$-variants are tested in combination with a slightly longer $5^{\prime}$-end (Fig. 3A, 166.X), resulting in a more stable P2, the effect of the length of the $3^{\prime}$-end is no longer apparent, all variants display a relatively high observed cleavage rate, and the reaction goes almost to completion (with the exception of the shortest version, 166.11). Taken together, these results support that the observed cleavage rates and end-points depend on the relative stabilities of the catalytically active structure with intact P2 and P10 and the inactive form with HEG P1 (Fig. 1).

Since the kinetic cleavage analyses do not discriminate between branching and hydrolysis, we performed primer extension analysis of all of the end-point samples from the analyses. Independent of the length of the 3 '-end, all versions containing $166 \mathrm{nt}$ upstream of IPS accumulate products of cleavage by hydrolysis at the IPS (Fig. 3A). Conversely, all the 157-nt variants accumulate products of cleavage by branching at the IPS (Fig. 3C). In the intermediate 162-nt variants, the reaction is dependent on the length of the $3{ }^{\prime}$-end. With a $3^{\prime}$-end of $\geq 37 \mathrm{nt}$, the products of cleavage by branching accumulate, whereas the shorter versions accumulate the products of cleavage by hydrolysis (Fig. 3B). Again, the shift in the outcome of the experiment with the 162-nt variants coincides with the potential to form HEG P1.

In summary, length variants with a very stable P2 (166.X) are independent of the length of the $3^{\prime}$-end and appear as a hydrolytic ribozyme. Shortening of P2 (162.X) results in ribozymes that are dependent on the length of the $3^{\prime}$-end, and the ability to form HEG P1 switches the ribozyme from a predominantly hydrolytic ribozyme in short variants to a branching ribozyme in longer variants. Finally, a relatively unstable P2 (157.X) makes the ribozyme appear as a branching ribozyme with an activity that strongly depends on the ability to form HEG P1. The variants fall in two discrete groups primarily defined by the end-points of cleavage. Variants that are able to form HEG P1 appear to misfold and cleave to a significantly lesser degree than those that are too short to form HEG P1. In all, it appears that several aspects of activity of the length variants of DiGIR1 can be explained by taking the relative stabilities of the active conformation characterized by the P2 and P10 elements and the inactive conformation characterized by HEG P1 into consideration.

\section{Addition of urea affects both the $\boldsymbol{k}_{\mathrm{obs}}$ and the resulting reaction}

With some of the length variants, cleavage by hydrolysis appears to be the dominant reaction, in contrast to the branching reaction that is the only reaction observed in vivo. Hydrolysis can be viewed as an alternative reaction in which a 
A
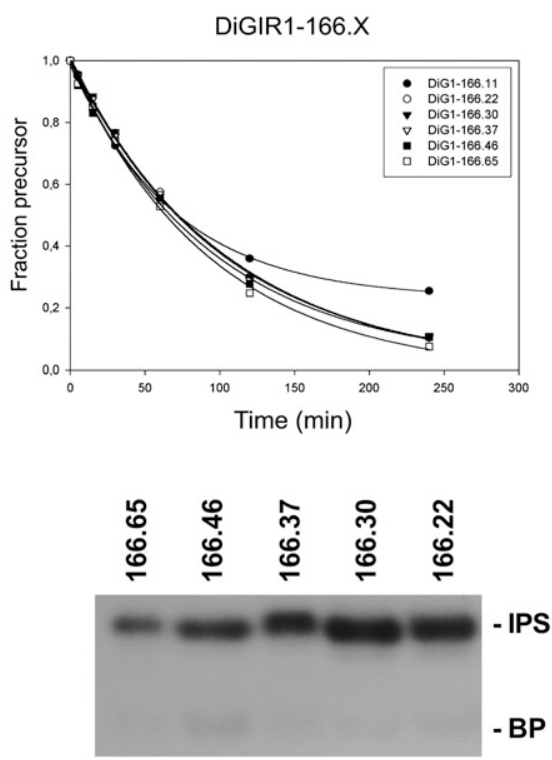

B
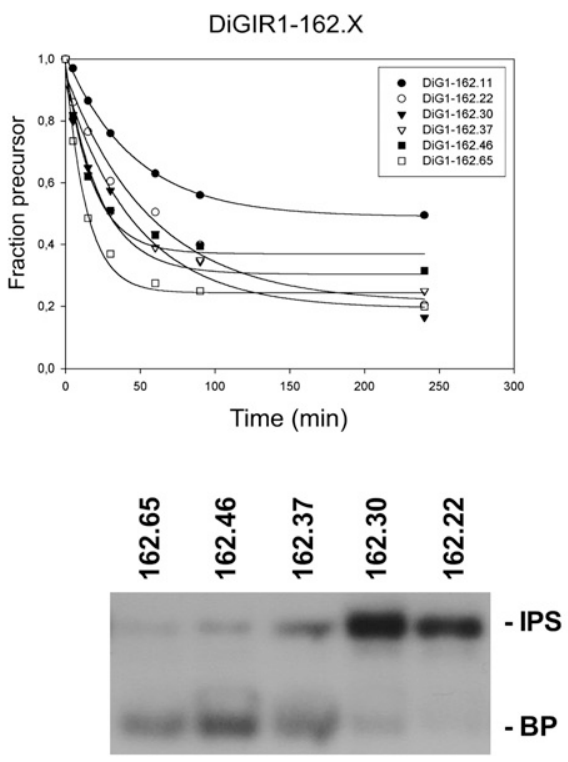

C
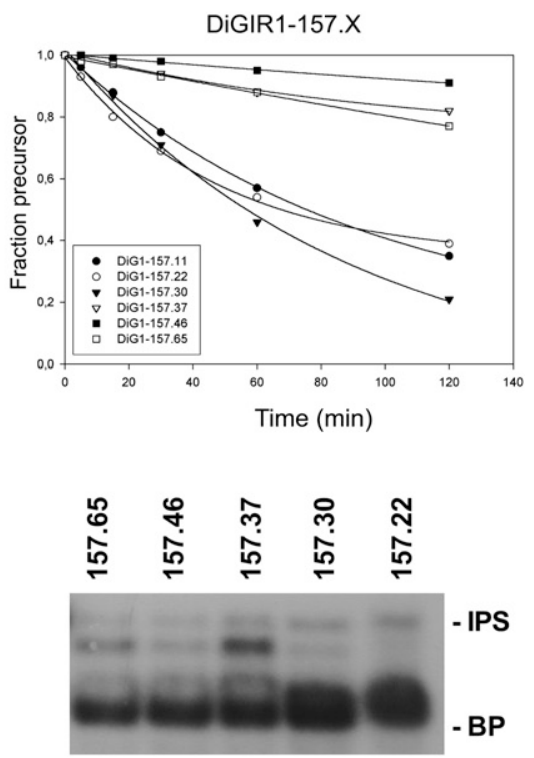

FIGURE 3. DiGIR1 cleavage and primer extension analyses of length variants of DiGIR1 with $166 \mathrm{nt}(A), 162 \mathrm{nt}(B)$, and $157 \mathrm{nt}(C)$ included upstream of the IPS. (Graphs) Cleavage analyses; (autoradiograms) primer extension analyses. Numbers refer to the positions indicated in Fig. 1. The figure in $B$ was constructed using data from Einvik et al. (2000).

water molecule acts as the attacking group in place of U232. The explanation for the dominance of this pathway in some length variants could be a folding problem resulting in a failure to present the nucleophilic 2'-OH of U232 properly at the reaction site in combination with a masking of the branching reaction by efficient reversal by ligation (Nielsen et al. 2005). To address this possibility, we have performed cleavage and ligation experiments in the presence of urea. Urea allows the ribozyme to search the conformational space, thus allowing a larger proportion of the molecules to be properly aligned and subsequently to undergo the branching reaction. A cleavage experiment of 162.22 in the presence of 2 $\mathrm{M}$ urea (Fig. 4) shows that urea has multiple effects on the reaction. The $k_{\mathrm{obs}}$ is increased sevenfold, and the reaction goes almost to completion within the experimental time ( 240 min) (Fig. 4A,B). In addition, the cleavage mechanism is shifted from hydrolysis toward branching as analyzed by primer extension (Fig. 4C). A similar shift in the cleavage rate and cleavage mechanism was observed in an experiment with 166.65 (data not shown). Similar to the effect of urea, an increase in $\mathrm{Mg}^{2+}$ concentration can shift variants of $162 . \mathrm{X}$ (e.g., 162.65) from branching toward hydrolysis (data not shown). Although these experiments demonstrate a clear effect on the reaction, they do not discriminate between effects on folding and ligation. A more direct experiment addressing the effect of urea on the ligation reaction was performed (Fig. 4D). Labeled (157)22 RNA (gel-purified 22nt 3 '-fragment from a branching reaction of 157.22) was mixed with unlabeled 166(22) in a time-course experiment. Considerably less ligated product accumulates over time in the presence of $2 \mathrm{M}$ urea. With this evidence at hand, we favor the idea that the effect of urea predominantly is inhibition of the ligation reaction rather than stimulation of cleavage. This implies that the outcome of the cleavage reaction (hydrolysis versus branching) is determined by the relative rates of branching and ligation (Fig. 2A).

\section{P2 and P10 become accessible to single-strand probes upon cleavage}

We next addressed the conformational change leading to formation of HEG P1 by studying the accessibility to chemical modification prior to and after cleavage. Kethoxal reacts with solvent-accessible $\mathrm{N} 1$ and $\mathrm{N} 2$ of guanosine, and the modifications are subsequently scored as pause signals in primer extension analysis. In a kethoxal modification experiment of DiGIR1 (Fig. 5A), the precursor 162.65 RNA (Pre) is protected against modification with kethoxal in stems P2, P2.1, and P10. In contrast, the $5^{\prime}$-strand of P2 and P10, but not the P2.1 stem, is modifiable by a kethoxal reaction performed directly in the cleavage reaction at late time-points when the cleavage reaction is at its end-point (162.65 RNA) (Fig. 5B, "Post"). This is consistent with the proposed conformational switch between P2 and P10 in the pre-cleavage conformation and HEG P1 in the postcleavage conformation. Similar accessibility was found with 166.65 (Post) RNA (Fig. 5B). This is surprising because this length variant cleaves by hydrolysis and without release of the $3^{\prime}$-product (see below). However, the result is consistent with the interpretation that the 166.65 variant carries out branching that is masked by efficient ligation of the products. Thus, 166.65 is envisaged to undergo cycles of 
A

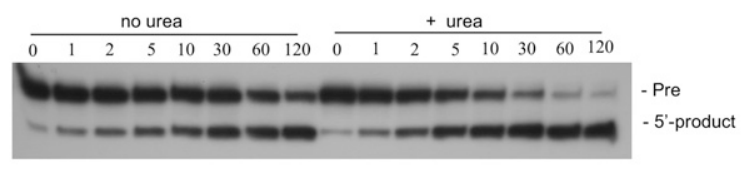

B

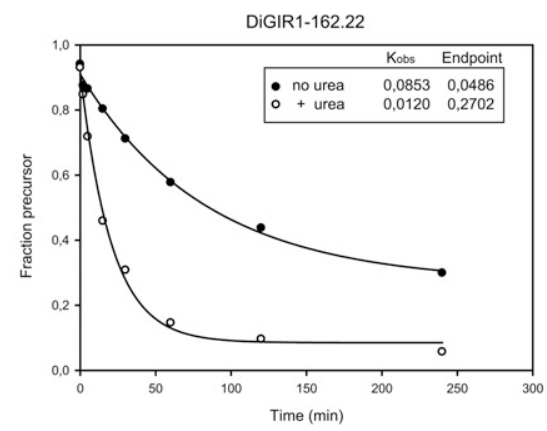

C

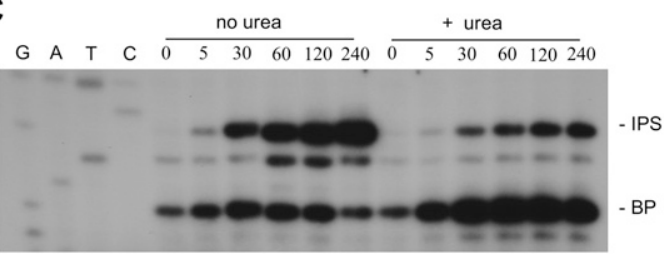

D

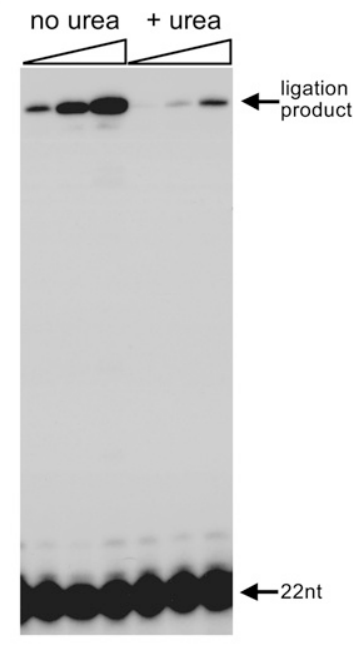

found to correlate with branching as evaluated by primer extension analysis. This observation supports a role of the cleavage reaction in downstream events relating to the fate of the I-DirI mRNA.

\section{DISCUSSION}

\section{The role of hairpin HEG P1 in DiGIR1 activity}

We have identified a conformational switching mechanism coupled to cleavage by transesterification in the DiGIR1 branching ribozyme. The mechanism was deduced from analysis of length variants and emphasizes the importance of the formation of the alternative hairpin HEG P1 both prior to and after cleavage. The variants with the longest $5^{\prime}$-end (the 166.X variants) were chosen because they are similar in reaction rate and outcome of the reaction to the full-length intron in vitro. They have a relatively stable catalytic core due to the presence of a long P2. However, the increased stability of P2 impairs the release of the products (Fig. 6), thereby promoting the reverse (ligation) reaction. The true forward rate is revealed only in the presence of urea that destabilizes P2 and furthermore inhibits ligation once the products are dissociated (Fig. 4). As a consequence, the reaction observed during standard cleavage conditions is the irreversible cleavage by

branching and ligation, interrupted by cleavage by hydrolysis that results in "dead-end" products. During the opening of P2-P10 in the branching phase, the P2' and P10' would be modifiable, as observed.

\section{Branching, but not hydrolysis, at IPS promotes release of the $3^{\prime}$-product}

One important problem with ribozyme-catalyzed cleavage and branching reactions is product release. In the case of DiGIR1, we have studied this problem by native gel electrophoresis of cleavage reactions. These experiments were carried out as standard cleavage experiments using gel-purified precursor RNA. The RNA was renatured and the reaction jumpstarted by increasing the $\mathrm{pH}$ from 5.5 to 7.5 . Time samples were withdrawn and split into aliquots for immediate loading onto native gels (Fig. 6A) or subsequent analysis on denaturing gels (Fig. 6B). Interestingly, release of the $3^{\prime}$-cleavage product was observed with 157.65 RNA and 162.65 RNA, but not with 166.65 RNA (Fig. 6A). Thus, the release of the $3^{\prime}$-product is hydrolysis. In the 162.X series, the catalytic core is less stable due to the shortening of P2. Because of this, the length of the $3^{\prime}$-end becomes important. The longer variants that are capable of forming HEG P1 have the highest $k_{\mathrm{obs}}$, and the primer extension analysis shows that branching is the dominant reaction. In the shorter variants, the reverse reaction becomes an important factor because HEG P1 no longer forms, and the $k_{\text {obs }}$ predominantly reflects the hydrolysis rate. Finally, in the 157.X series, P2 is shortened to the extent that very little ligation occurs. The $k_{\text {obs }}$ reflects the branching reaction only and is relatively low due to the unstable core; furthermore, it is dependent on the length of the 3 '-fragment. In the long variants that are capable of forming HEG P1, the activity is very low. This is due to the increased relative stability of the inactive conformation that includes HEG P1 compared with the catalytically active conformation that includes P2.

The hairpin HEG P1 was originally proposed to be a structural component of the $5^{\prime}$-UTR of the I-DirI mRNA (Vader et al. 1999). Indeed, this appears to be the case, as 
A

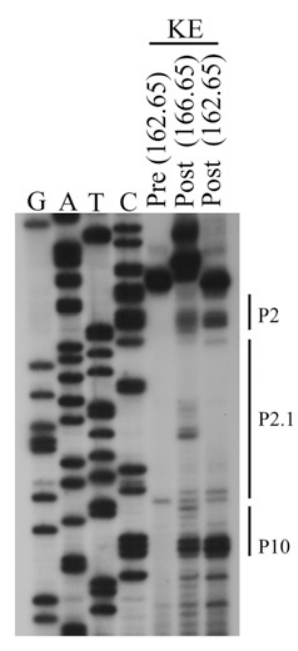

B
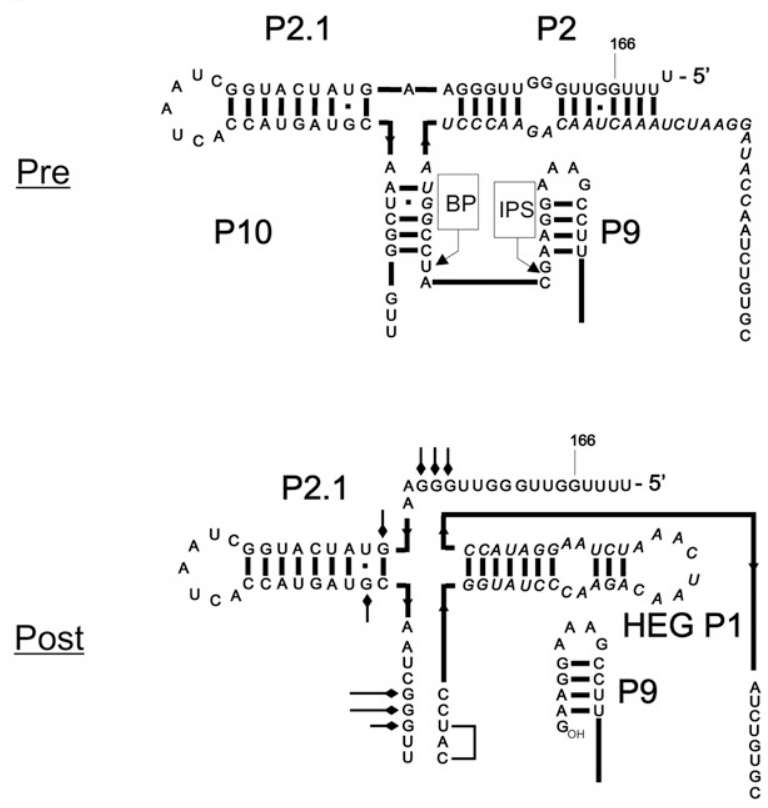

FIGURE 5. Accessibility of structural elements $\mathrm{P} 2, \mathrm{P} 2.1$, and $\mathrm{P} 10$ to kethoxal modification prior to and after cleavage. (A) Primer extension analysis of modified RNA prior to cleavage (Pre [162.65]) and after cleavage by hydrolysis (Post [166.65]) or branching (Post [162.65]). A sequencing ladder made by sequencing of a DiGIR1 plasmid with the same primer as used for primer extension is shown to the left. (B) Helix diagrams showing the structure of the P2-P2.1P10 domain prior to (Pre) and after (Post) cleavage. The nucleotides involved in forming HEG $\mathrm{P} 1$ are italicized. The observed modifications are indicated in the post-cleavage structure. None of these structures (indicated by arrows) were found to be modified in the precursor RNA (Pre).

evidenced by structure probing and modeling of the mRNA (M. Hedegaard and H. Nielsen, unpubl.). The present study and other data suggest multiple roles of HEG P1 in upstream processes. First, the formation of HEG P1 can inhibit DiGIR1 cleavage in some length variants. This is consistent with structure probing data that identify HEG P1 as a component of an inactive conformation of DiGIR1 that forms upon transcription of full-length intron with flanking exons (M. Hedegaard and H. Nielsen, unpubl.). Second, HEG P1 is formed following cleavage by transesterification, and its formation plays a role in driving this reaction (see below). Finally, the internal loop of HEG P1 is a tetraloop receptor that is involved in a post-cleavage tertiary interaction with DiGIR1 (A. Birgisdottir and S.D. Johansen, unpubl.). Thus, it appears that HEG P1 mediates an intimate linkage between the DiGIR1 ribozyme and the I-DirI mRNA and is a key element in regulating the activities of these two components of twin-ribozyme introns.

Future effort should be directed toward isolating the individual reactions catalyzed by DiGIR1. In the present study, the branching reaction was assessed based on cleavage in the presence of $2 \mathrm{M}$ urea, because it was found that these conditions inhibit the reverse reaction. The reverse (ligation) reaction was assessed in acidic cleavage buffer, because the forward reaction is inhibited under these conditions (Johansen and Vogt 1994; Einvik et al. 1998). No attempts were made to isolate the hydrolytic cleavage reaction, but it was inferred that the cleavage rate of the 166.X series reflected this rate. In the present study, the highest $k_{\text {obs }}$ that could be taken to represent the branching reaction is $0.085 \mathrm{~min}^{-1}$ for the 162.22 variant in the presence of $2 \mathrm{M}$ urea. This is only one order of magnitude less than the cleavage rates of most optimized minimal cleavage ribozymes (e.g., $0.2-0.5$ $\mathrm{min}^{-1}$ for the hairpin [Fedor 2000], $1.0 \mathrm{~min}^{-1}$ for the VS [Lafontaine et al. 2001], and 0.5-2.0 $\mathrm{min}^{-1}$ for the hammerhead ribozyme [Clouet-d'Orval and Uhlenbeck 1997]). In evaluating such rates, it should be kept in mind that low rates do not necessarily reflect a deficiency because these ribozymes evolved as single-turnover catalysts. Also, the true rate in many cases has been shown to be much higher when more complete versions of the ribozymes have been studied. Examples are the hammerhead ribozyme that has a 50-fold higher rate when a peripheral domain is included (Peracchi et al. 1998; Khvorova et al. 2003) and the VS ribozyme (Zamel et al. 2004). In the case of DiGIR1, the requirement for high salt concentrations in vitro could indicate a requirement for a protein factor, although this could also be an artifact of the lack of proper sequence context. However, the present study shows that there is no reason to assume that such a factor would be necessary to stabilize the core. Alternatively, it could be a helicase involved in destabilization of the inactive conformation of DiGIR1 or in assisting the dissociation of the products.

\section{The conformational switch}

Product release is a rate-limiting step for many cleavage and transesterification ribozymes in vitro (Cech and Golden 1999). Following cleavage at the IPS, the I-DirI mRNA is potentially bound to the DiGIR1 core ribozyme by 19 base pairs (bp) and several tertiary interactions. A structural transition into a HEG P1-containing structure compensates by the formation of $10 \mathrm{bp}$. However, a full comparison of the energy conservation of the transition is not possible. The tertiary interactions in the two structures have not been fully worked out, and the structure of the GU-rich $5^{\prime}$-strands of P10 and P2 following cleavage has not been determined. Furthermore, it is important to keep in mind that the biologically relevant reaction takes place in the context of the 
A

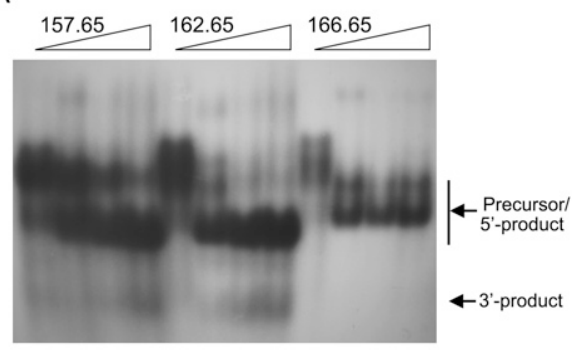

B

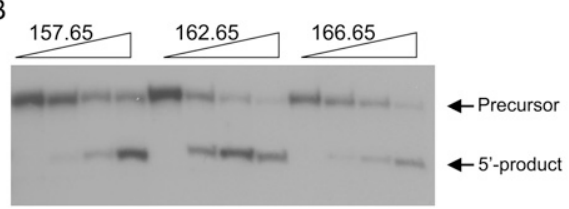

FIGURE 6. Cleavage analysis of three different $5^{\prime}$-length variants analyzed in parallel on native $(A)$ and $6 \%$ denaturing (urea) polyacrylamide $(B)$ gels. The cleavage reactions were incubated for $0,5,30$, and $120 \mathrm{~min}$, respectively. The $5^{\prime}$ - and $3^{\prime}$-products are indicated.

full-length intron. With respect to bonding energy, the branching reaction results in the replacement of a $3^{\prime}, 5^{\prime}$ phosphodiester bond with a $2^{\prime}, 5^{\prime}$-bond contained in a tiny lariat that has a constrained conformation (Agback et al. 1993). It appears that the structural transition is triggered in vitro if the cleavage occurs by branching but not if the cleavage is by hydrolysis (Fig. 6). It is possible that the constraints of the lariat initiate the transition by breaking the basal base pairs of P10 and possibly by releasing a tertiary interaction involving P10. In this way, the transition is a kind of "zippering" initiated by the branching reaction. It cannot be ruled out that the reaction in vivo requires a protein factor, e.g., in the form of a helicase. If this was the case, it would require adaptation to the host because no such helicase activity is encoded by the intron.

The disruption and subsequent re-formation of HEG P1 in relation to DiGIR1 activity is reminiscent of the relation of P-1 to the group I intron in Tetrahymena (Fig. 7A; Emerick and Woodson 1993). P-1 is a cotranscriptionally favored hairpin located in the $5^{\prime}$-exon that competes with P1 harboring the $5^{\prime}$-splice site with the additional consequence of misfolding of the core (Pan and Woodson 1998). Thus, the formation of P-1 initially results in down-regulation of splicing just like the initial, cotranscriptionally favored formation of HEG P1 results in an inactive DiGIR1. Once the transcript is extended into the $3^{\prime}$-exon, sequences become available that result in a structure that will disrupt P-1 by including its $5^{\prime}$-strand (Emerick and Woodson 1993; Cao and Woodson 1998, 2000). Now, P1 is formed and the intron is spliced out. A similar "trigger" sequence for the conformational switching has not been identified in DiGIR1, but overall this step corresponds to the disruption of HEG P1 by formation of the catalytically active core and sub- sequent branching. In the Tetrahymena case, $\mathrm{P}-1$ is now reformed by a conformational switching that results in release of the spliced out intron (Cao and Woodson 2000). This is obviously paralleled by the coupled release of the I-DirI mRNA and formation of HEG P1. Finally, P-1 is found as part of the mature rRNA (Cao and Woodson 2000), just like HEG P1 is found as part of the mature I-DirI mRNA. The conformational switching described in the present paper thus illustrates what might very well be a general principle in RNA-directed RNA processing.

\section{The function of DiGIR1 is to promote expression of the I-Dirl mRNA}

DiGIR1 is an example of a ribozyme with a derived function. It has long been known that DiGIR1 is required

A

\begin{tabular}{ll} 
Tetrahymena intron splicing & Didymium GIR1 branching \\
\hline $\begin{array}{l}\text { Formation of } \mathrm{P}-1 \text { inhibits } \\
\mathrm{P} 1 \text { formation and splicing }\end{array}$ & $\begin{array}{l}\text { Formation of HEG P1 inhibits } \\
\mathrm{P} 10-\mathrm{P} 2 \text { formation and branching }\end{array}$ \\
\hline $\begin{array}{l}\mathrm{P} 1 \text { formation is triggered by } \\
\text { transcription of downstream } \\
\text { sequences that disrupt } \mathrm{P}-1\end{array}$ & $\begin{array}{l}\mathrm{P} 10-\mathrm{P} 2 \text { formation is triggered by } \\
\text { completion of transcription of the } \\
\text { intron followed by splicing }\end{array}$ \\
\hline $\begin{array}{l}\mathrm{P}-1 \text { is reformed resulting in } \\
\text { the release of the intron }\end{array}$ & $\begin{array}{l}\text { HEG P1 is reformed resulting in } \\
\text { the release of the mRNA }\end{array}$ \\
\hline $\begin{array}{l}\mathrm{P}-1 \text { is found as part of the } \\
\text { mature rRNA }\end{array}$ & HEG P1 is found as part of the \\
\hline
\end{tabular}

B

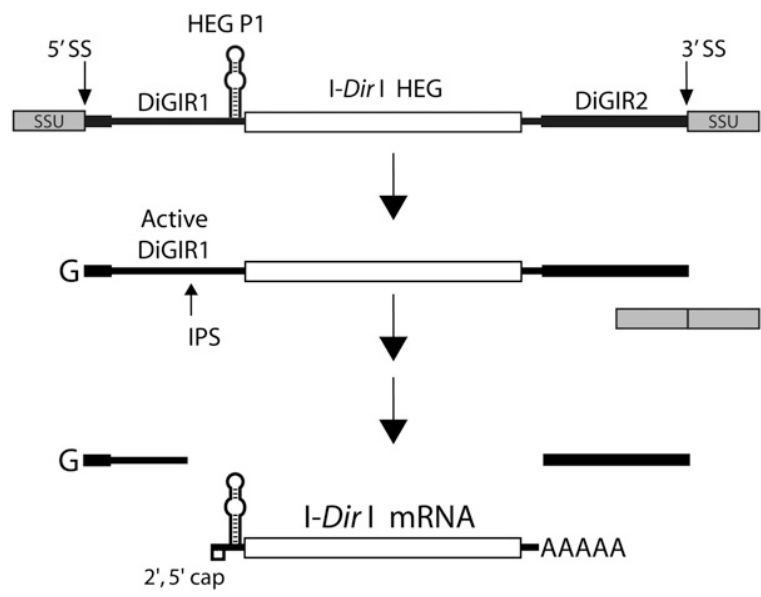

FIGURE 7. (A) Comparison of features of conformational change between the Tetrahymena group I splicing ribozyme and the Didymium GIR1 branching ribozyme. (B) Schematic outline of the conformational switch involved in I-DirI mRNA maturation. In the precursor transcript (top), HEG P1 is formed, resulting in an inactive DiGIR1 ribozyme. After DiGIR2 catalyzed self-splicing, a conformational switch reorganizes the HEG P1 residues into the active form of DiGIR1 with subsequent cleavage by branching at the internal processing site (IPS). HEG P1 then reappears in the mature I-DirI mRNA (bottom) along with a lariat cap and a poly $(A)$ tail at the $5^{\prime}$ - and $3^{\prime}$-ends, respectively. (SSU) Small subunit ribosomal RNA exon, $\left(5^{\prime}\right.$ - and $3^{\prime}-$-SS $) 5^{\prime}$ - and $3^{\prime}$-splice sites, $(G)$ exogenous guanosine cofactor added at the $5^{\prime}$-end of the excised intron RNA during self-splicing. 
for the formation of the $5^{\prime}$-end of the I-DirI mRNA (Fig. 7B). It now appears that DiGIR1 is involved in the promotion of I-DirI mRNA expression in multiple ways. First, it delays the processing of the mRNA by adopting an inactive conformation until the intron is released from the rRNA precursor by splicing. Second, it provides the mRNA with a cap in the form of a tiny lariat. This cap protects the mRNA against $5^{\prime}-3^{\prime}$ exonucleases. It is not known if the lariat cap can substitute for other functions of a conventional $m e^{7} \mathrm{G}$ cap, e.g., in translation initiation. Third, it undergoes a conformational change that results in the release of the mRNA $5^{\prime}$-end from the core ribozyme. Fourth, in doing so, it promotes the formation of a structured $5^{\prime}$-end of the mRNA. And fifth, it becomes re-associated with the mRNA through a tertiary interaction involving a tetraloop receptor in the $5^{\prime}$-UTR (A. Birgisdottir and S.D. Johansen, unpubl.). In conclusion, DiGIR1 is an example of a group I ribozyme that has become adopted to facilitate the expression of a protein-encoding gene in multiple ways.

\section{MATERIALS AND METHODS}

\section{Oligonucleotides, templates, and in vitro transcription}

Templates for in vitro transcription were made by PCR using Pfu DNA polymerase as previously described (Einvik et al. 2000). In length variant experiments, the 5 '-primers (all including a T7 RNA polymerase promoter) were:

\section{C289: AATTTAATACGACTCACTATAGGTTGGGTTGGGAAGT ATCAT (DiGIR1-166); \\ C287: AATTTAATACGACTCACTATAGGGTTGGGAAGTATCAT (DiGIR1-162); and \\ C294: AATTTAATACGACTCACTATAGGGAAGTATCAT (DiGI R1-157). \\ The 3 '-oligos used to include a variable number of nucleotides downstream from IPS were: \\ OP78: GATACCGGATGCTTCCTT (11 nt); OP233: GATTGTCTTGGGATACCG (22 nt); \\ OP353: TTAGATTTGATTGTCTTG (30 nt); \\ OP314: GGTATCCTTAGATTTGATTGTCTT (37 nt); \\ OP235: GCACAGATTGGTATCCTT (46 nt); and \\ OP12: TCACCATGGTTGTTGAAGTGCACAGATTG (65 nt). \\ The $3^{\prime}$-oligos used to make templates with mutations in the struc- tural element HEG P1 were: \\ OP878: TCACCATGGTTGTTGAAGTGCACAGATTGGTATCCG GAGATTTGATTGTCTTGGGAT (HEG P1mut1); and OP879: TCACCATGGTTGTTGAAGTGCACAGATTTCATAGGA ATCTTTTGATTGTCTTGGGATACC (HEG P1mut2).}

All templates were transcribed by in vitro transcription using T7 RNA polymerase (Fermentas) using trace amounts of $\left[\alpha-{ }^{32} \mathrm{P}\right] \mathrm{UTP}$ as a label as previously described (Einvik et al. 2000).

\section{Cleavage experiment and quantitative analysis}

The cleavage analysis was performed as described by Einvik et al. (2000). Briefly, radioactively labeled in vitro transcripts made from PCR-templates and renatured in $1 \mathrm{M} \mathrm{KCl}, 25 \mathrm{mM} \mathrm{MgCl}_{2}$ at $\mathrm{pH} 5.5$ for $10 \mathrm{~min}$ at $45^{\circ} \mathrm{C}$. Then, the reaction was jump-started by increasing the $\mathrm{pH}$ to 7.5 by addition of Hepes-KOH. Timesamples were withdrawn and run on $6 \%$ denaturing (urea) polyacrylamide gels. The gels were analyzed on storage phosphor screens, and the data were fitted to a nonlinear first-order decay equation. All transcripts were analyzed at least three times, and the results were highly reproducible.

\section{Primer extension analysis}

Primer extension analyses were carried out as previously described (Einvik et al. 2000) using the following end-labeled oligos: C291: 5'-GATTGTCTTGGGAT or C301: 5'-TCACCATGGTTGTTGA (depending on the particular length variant) for analysis of cleavage and ligation reactions, and C80: 5'-ATCGTCCCCCACC GTT for analysis of the chemical modification reactions. Sequencing ladders were made using the same primers and the plasmid pDi162G1 (Einvik et al. 2000). The reactions were analyzed on $8 \%$ denaturing (urea) polyacrylamide gels. The primer extension stop signals were previously referred to as IPS1 and IPS2 (Einvik et al. 1998, 2000) but are renamed IPS (internal processing site; the cleavage site) and BP (branch point), respectively, in this study in accordance with the characterization of the reaction as a branching reaction (Nielsen et al. 2005).

\section{Ligation experiments}

RNA for ligation experiments was gel purified as described (Kjems et al. 1998) from standard cleavage reactions. The $5^{\prime}$-fragment was unlabeled and the $3^{\prime}$-fragment radioactively labeled during transcription. The $5^{\prime}$-fragment was renatured for $10 \mathrm{~min}$ at $45^{\circ} \mathrm{C}$ in $1 \mathrm{M} \mathrm{KCl}, 25 \mathrm{mM} \mathrm{MgCl}_{2}, 10 \mathrm{mM} \mathrm{K}$-Acetate at $\mathrm{pH} 5.5$ before addition of a molar excess of the $3^{\prime}$-fragment in $1 \mathrm{M} \mathrm{KCl}, 25 \mathrm{mM}$ $\mathrm{MgCl}_{2}, 47.5 \mathrm{mM}$ Hepes- $\mathrm{KOH}$ at $\mathrm{pH} 7.5$, and samples were withdrawn at the time points indicated for subsequent analysis on $8 \%$ denaturing (urea) polyacrylamide gels. For the experiment using 3 '-fragments with mutations in HEG P1, the ligation reaction was carried out in acidic renaturation buffer in order to inhibit the forward reaction.

\section{Chemical modification}

Kethoxal modification was carried out as described (Kjems et al. 1998). The precursor RNA was transcribed from a 162.65 template and probed in renaturation buffer. The cleaved RNA was incubated in cleavage buffer for $120 \mathrm{~min}$. At this time point, $>85 \%$ of the precursor was cleaved as analyzed by gel electrophoresis. The modified RNA was analyzed by primer extension using primer $\mathrm{C} 80$ as described above.

\section{Native gel analysis}

Native gels were $6 \%$ polyacrylamide gels electrophoresed in a running buffer (34 mM Tris- $\mathrm{HCl}$ at $\mathrm{pH} 7.5,68 \mathrm{mM}$ Hepes- $\mathrm{KOH}$ at $\mathrm{pH} 7.5,0.1 \mathrm{mM}$ EDTA, $10 \mathrm{mM}$ EDTA) at $4^{\circ} \mathrm{C}$ as described (Emerick and Woodson 1994). The samples for cleavage analysis were treated essentially as described above. However, to allow simultaneous loading onto the gel, the reactions were initiated at various time points $(t=0,5,30$, and $120 \mathrm{~min})$ prior to loading on the gel. 


\section{SUPPLEMENTAL MATERIAL}

Supplemental material can be found at http://www.rnajournal.org.

\section{ACKNOWLEDGMENTS}

The work was supported by grants from The Danish Natural Science Research Council (H.N.), The Carlsberg Foundation (H.N.), The NOVO Nordic Foundation (H.N.), The Lundbeck Foundation (H.N.), The Norwegian Research Council (S.D.J.), and The Norwegian Cancer Society (S.D.J.). T.E.L. was supported by a fellowship from The Danish Medical Research Council.

Received June 1, 2008; accepted January 23, 2009.

\section{REFERENCES}

Agback, P., Sandstrom, A., Yamakage, S., Sund, C., Glemarec, C., and Chattopadhyaya, J. 1993. Solution structure of lariat RNA by 500 MHz NMR spectroscopy and molecular dynamics studies in water. J. Biochem. Biophys. Methods 27: 229-259.

Badorrek, C.S., Gherghe, C.M., and Weeks, K.M. 2006. Structure of an RNA switch that enforces stringent retroviral genomic RNA dimerization. Proc. Natl. Acad. Sci. 103: 13640-13645.

Beckert, B., Nielsen, H., Einvik, C., Johansen, S.D., Westhof, E., and Masquida, B. 2008. Molecular modelling of the GIR1 branching ribozyme gives new insight into evolution of structurally related ribozymes. EMBO J. 27: 667-678.

Cao, Y. and Woodson, S.A. 1998. Destabilizing effect of an rRNA stem-loop on an attenuator hairpin in the $5^{\prime}$ exon of the Tetrahymena pre-rRNA. RNA 4: 901-914.

Cao, Y. and Woodson, S.A. 2000. Refolding of rRNA exons enhances dissociation of the Tetrahymena intron. RNA 6: 1248-1256.

Cech, T.R. and Golden, B.L. 1999. Building a catalytic active site using only RNA. In The RNA world (eds. R.F. Gesteland et al.), pp. 321349. Cold Spring Harbor Laboratory Press, Cold Spring Harbor, New York.

Clouet-d'Orval, B. and Uhlenbeck, O.C. 1997. Hammerhead ribozymes with a faster cleavage rate. Biochemistry 36: 9087-9092.

Decatur, W.A., Einvik, C., Johansen, S., and Vogt, V.M. 1995. Two group I ribozymes with different functions in a nuclear rDNA intron. EMBO J. 14: 4558-4568.

Decatur, W.A., Johansen, S., and Vogt, V.M. 2000. Expression of the Naegleria intron endonuclease is dependent on a functional group I self-cleaving ribozyme. RNA 6: 616-627.

Einvik, C., Nielsen, H., Westhof, E., Michel, F., and Johansen, S. 1998. Group I-like ribozymes with a novel core organization perform obligate sequential hydrolytic cleavages at two processing sites. RNA 4: 530-541.

Einvik, C., Nielsen, H., Nour, R., and Johansen, S. 2000. Flanking sequences with an essential role in hydrolysis of a self-sleaving group I-like ribozyme. Nucleic Acids Res. 28: 2194-2200.

Emerick, V.L. and Woodson, S.A. 1993. Self-splicing of the Tetrahymena pre-rRNA is decreased by misfolding during transcription. Biochemistry 32: 14062-14067.

Emerick, V.L. and Woodson, S.A. 1994. Fingerprinting the folding of a group I precursor RNA. Proc. Natl. Acad. Sci. 91: 9675-9679.
Fedor, M.J. 2000. Structure and function of the hairpin ribozyme. J. Mol. Biol. 297: 269-291.

Johansen, S. and Vogt, V.M. 1994. An intron in the nuclear ribosomal DNA of Didymium iridis codes for a group I ribozyme and a novel ribozyme that cooperate in self-splicing. Cell 76: 725-734.

Johansen, S., Einvik, C., and Nielsen, H. 2002. DiGIR1 and NaGIR1: Naturally occurring group I-like ribozymes with unique core organization and evolved biological role. Biochimie 84: 905-912.

Johansen, S.D., Haugen, P., and Nielsen, H. 2007. Expression of protein-coding genes embedded in ribosomal DNA. Biol. Chem. 388: 679-686.

Khvorova, A., Lescoute, A., Westhof, E., and Jayasena, S.D. 2003. Sequence elements outside the hammerhead ribozyme catalytic core enable intracellular activity. Nat. Struct. Biol. 10: 708-712.

Kjems, J., Egebjerg, J., and Christiansen, J. 1998. Analysis of RNAprotein complexes in vitro. Elsevier, Amsterdam.

Konarska, M.M., Vilardell, J., and Query, C.C. 2006. Repositioning of the reaction intermediate within the catalytic center of the spliceosome. Mol. Cell 21: 543-553.

Lafontaine, D.A., Wilson, T.J., Norman, D.G., and Lilley, D.M. 2001. The A730 loop is an important component of the active site of the VS ribozyme. J. Mol. Biol. 312: 663-674.

Nagel, J.H. and Pleij, C.W. 2002. Self-induced structural switches in RNA. Biochimie 84: 913-923.

Nagel, J.H., Gultyaev, A.P., Oistämö, K.J., Gerdes, K., and Pleij, C.W. 2002. A pH-jump approach for investigating secondary structure refolding kinetics in RNA. Nucleic Acids Res. 30: e63. doi: 10.1093/ nar/gnf057.

Nielsen, H., Westhof, E., and Johansen, S. 2005. An mRNA is capped by a $2^{\prime}, 5^{\prime}$ lariat catalyzed by a group I-like ribozyme. Science 309: $1584-1587$.

Nielsen, H., Beckert, B., Masquida, B., and Johansen, S.D. 2008. The GIR1 branching ribozyme. In Ribozymes and RNA catalysis (eds. D.M. Lilley and F. Eckstein), pp. 229-252. RSC Publishing, Cambridge, UK.

Pan, J. and Woodson, S.A. 1998. Folding intermediates of a self-splicing RNA: Mispairing of the catalytic core. J. Mol. Biol. 280: 597-609.

Peracchi, A., Karpeisky, A., Maloney, L., Beigelman, L., and Herschlag, D. 1998. A core folding model for catalysis by the hammerhead ribozyme accounts for its extraordinary sensitivity to abasic mutations. Biochemistry 37: 14765-14775.

Rodnina, M.V. and Wintermeyer, W. 2003. Peptide bond formation on the ribosome: Structure and mechanism. Curr. Opin. Struct. Biol. 13: 334-340.

Schroeder, R., Barta, A., and Semrad, K. 2004. Strategies for RNA folding and assembly. Nat. Rev. Mol. Cell Biol. 5: 908-919.

Thirumalai, D., Lee, N., Woodson, S.A., and Klimov, D. 2001. Early events in RNA folding. Annu. Rev. Phys. Chem. 52: 751-762.

Tucker, B.J. and Breaker, R.R. 2005. Riboswitches as versatile gene control elements. Curr. Opin. Struct. Biol. 15: 342-348.

Vader, A., Nielsen, H., and Johansen, S. 1999. In vivo expression of the nucleolar group I intron-encoded I-DirI homing endonuclease involves the removal of a spliceosomal intron. EMBO J. 18: 1003-1013.

Woodson, S.A. 2002. Folding mechanisms of group I ribozymes: Role of stability and contact order. Biochem. Soc. Trans. 30: 1166-1169.

Zamel, R., Poon, A., Jaikaran, D., Andersen, A., Olive, J., De, A.D., and Collins, R.A. 2004. Exceptionally fast self-cleavage by a Neurospora Varkud satellite ribozyme. Proc. Natl. Acad. Sci. 101: 1467-1472. 

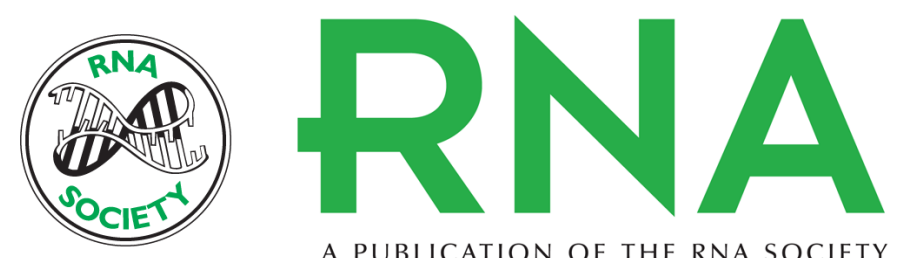

A PUBLICATION OF THE RNA SOCIETY

\section{A conformational switch in the DiGIR1 ribozyme involved in release and folding of the downstream I- Dir mRNA}

Henrik Nielsen, Christer Einvik, Thomas E. Lentz, et al.

RNA 2009 15: 958-967 originally published online March 27, 2009

Access the most recent version at doi:10.1261/rna.669209

\section{Supplemental http://rnajournal.cshlp.org/content/suppl/2009/03/30/rna.669209.DC1 \\ Material}

References This article cites 31 articles, 9 of which can be accessed free at: http://rnajournal.cshlp.org/content/15/5/958.full.html\#ref-list-1

\section{License}

Email Alerting Receive free email alerts when new articles cite this article - sign up in the box at the Service top right corner of the article or click here.

To subscribe to $R N A$ go to:

http://rnajournal.cshlp.org/subscriptions 